\title{
Spectroscopic ellipsometry of composite thin films with embedded Bi nanocrystals
}

\author{
R. Serna ${ }^{\text {a) }}$ \\ Instituto de Optica, CSIC, Serrano 121, 28006 Madrid, Spain \\ J. C. G. de Sande \\ Departamento de Ingenieria de Circuitos y Sistemas, E.U.I.T.T., U.P.M., Ctra. de Valencia km 7.5, \\ 28031 Madrid, Spain
}

J. M. Ballesteros and C. N. Afonso

Instituto de Optica, CSIC, Serrano 121, 28006 Madrid, Spain

(Received 1 December 1997; accepted for publication 7 July 1998)

\begin{abstract}
Spectroscopic ellipsometry together with an effective medium model is used to determine simultaneously the effective refractive index, thickness, and metal volume fraction of thin nanocomposite films. The films are formed by Bi nanocrystals embedded in amorphous matrices, either semiconducting $(\mathrm{Ge})$ or dielectric $\left(\mathrm{Al}_{2} \mathrm{O}_{3}\right)$. For the $\mathrm{Bi}: \mathrm{Ge}$ films (metal in an absorbing host), the values obtained for both the real and imaginary parts of the refractive index vary continuously from that of $\mathrm{Ge}$ to that of $\mathrm{Bi}$. The metal contents determined from the ellipsometry analysis are in excellent agreement with those obtained from direct measurements of the composition. For the $\mathrm{Bi}: \mathrm{Al}_{2} \mathrm{O}_{3}$ films (metal in a nonabsorbing host), the extinction coefficient $(k)$ exhibits a maximum around $360 \mathrm{~nm}$ which is related to the metal plasmon resonance frequency of Bi nanocrystals. The metal content determined from the ellipsometry analysis in this case is underestimated, probably due to interaction of the $\mathrm{Bi}$ crystals with the $\mathrm{Al}_{2} \mathrm{O}_{3}$ host. (C) 1998 American Institute of Physics. [S0021-8979(98)07619-1]
\end{abstract}

\section{INTRODUCTION}

Composite films are new artificially engineered materials with improved chemical, mechanical, magnetic, or optical properties. Those formed by metallic nanocrystals embedded in dielectric matrices have received much attention due to their specific optical absorption (colored filter glasses), and more recently due to the large enhancement of the intrinsic electronic nonlinear susceptibility which makes them potential candidates for integrated all-optical devices. ${ }^{1,2}$ Although the most studied metals have been $\mathrm{Au}, \mathrm{Cu}$, and $\mathrm{Ag}$, an enhancement of the nonlinear optical properties has recently been reported for oxide glasses containing heavy metals such as $\mathrm{Sb}$ and $\mathrm{Bi}^{3}{ }^{3}$ Nevertheless, the broad application of these kinds of compound systems requires the development of reliable techniques both to produce them as thin films and to characterize their optical properties, composition, and structure.

Pulsed laser deposition (PLD) has been reported as a suitable technique to produce such nanocrystalline composites, however, to our knowledge, its use has been very limited and mainly restricted to produce semiconductor nanocrystals by deposition in a gas environment. ${ }^{4,5}$ Recently, the growth of thin films with metallic $(\mathrm{Cu})$ nanocrystals embedded in $\mathrm{Al}_{2} \mathrm{O}_{3}$ host by PLD in vacuum has been demonstrated. Moreover, these films exhibit a large third-order nonlinear susceptibility $\left(\chi^{(3)}\right)$ value. $^{6}$

The linear optical properties of metal composite thin films have generally been studied by means of standard re-

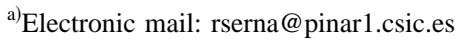

flection or transmission measurements over a wide wavelength range. ${ }^{7-9}$ Spectroscopic ellipsometry (SE) is a technique that has unquestionable advantages when compared to the usual reflectometry measurements. First, two parameters instead of one are independently determined in any singlemeasurement operation. Consequently, both real and imaginary parts of the complex refractive index $(\mathbf{n}=n+i k)$ of an homogeneous material can directly be obtained on a wavelength-by-wavelength basis. Second, SE measurements are relatively insensitive to intensity fluctuations of the source, temperature drifts of electronic components, and macroscopic roughness. ${ }^{10}$ Furthermore, the combination of $\mathrm{SE}$ with an effective medium model has been proved to be a successful means to estimate the stoichiometry of oxide thin films such as $\mathrm{SbO}_{x}$ and $\mathrm{GeO}_{x} \cdot{ }^{11,12}$ In spite of these favorable features together with its noninvasive character, the potentials of SE to the analysis of nanocrystalline composite thin films have not been explored so far.

In this article, we report the optical characterization by $\mathrm{SE}$ of composite films with $\mathrm{Bi}$ nanocrystals in $\mathrm{Ge}$ (Bi:Ge films) and $\mathrm{Al}_{2} \mathrm{O}_{3}\left(\mathrm{Bi}: \mathrm{Al}_{2} \mathrm{O}_{3}\right.$ films) hosts produced by PLD in vacuum. The $\mathrm{Bi}$ and $\mathrm{Ge}$ form a eutectic system with low mutual solubility, even in the liquid phase and no metastable alloy has been found. ${ }^{13}$ The low mutual solubility of the elements will ensure that all the deposited $\mathrm{Bi}$ will tend to form pure isolated and unreacted $\mathrm{Bi}$ crystals protected against atmospheric oxidation by the Ge matrix. The ellipsometric parameters are determined in a wide spectral range and their simulation by means of an effective medium model will allow us to estimate the metal content of the composite 
film. The comparison of the results in the Bi:Ge system to those obtained in the $\mathrm{Bi}: \mathrm{Al}_{2} \mathrm{O}_{3}$ system, in which the host is an oxide which can interact with the metal, will allow us to discuss not only the influence of possible interactions but also the validity of the method to determine the metal content in composite films in which the matrix is absorbing $(\mathrm{Ge})$ or nonabsorbing $\left(\mathrm{Al}_{2} \mathrm{O}_{3}\right)$. The latter host has, in addition to its wide transparency range, excellent thermal properties and has been reported to be an excellent host to produce nanocomposite films with large third-order nonlinear susceptibility. ${ }^{6}$

\section{EXPERIMENT}

PLD was carried out by focusing an ArF laser (12 ns, $193 \mathrm{~nm})$ at $5 \mathrm{~Hz}$ onto the rotating targets, at an incidence angle of $45^{\circ}$, and with an energy density of $2 \mathrm{~J} / \mathrm{cm}^{2}$. The experiments were carried out in vacuum at a base pressure of $7 \times 10^{-7}$ Torr. The substrate-target distance was $32 \mathrm{~mm}$. For the growth of the $\mathrm{Bi}: \mathrm{Ge}$ and $\mathrm{Bi}: \mathrm{Al}_{2} \mathrm{O}_{3}$ films, pure $\mathrm{Bi}$ and either $\mathrm{Ge}$ or $\mathrm{Al}_{2} \mathrm{O}_{3}$ targets were mounted on a computer controlled multiple holder which allowed to expose each of them alternately to the laser beam. The films were grown according to the following deposition sequence: a number of pulses on the $\mathrm{Bi}$ target, and then on the $\mathrm{Ge}$ or $\mathrm{Al}_{2} \mathrm{O}_{3}$ targets. This procedure was then repeated a fixed number of times. The $\mathrm{Bi}$ was always deposited first, and the matrix element was the last one deposited in order to protect the Bi crystals against atmospheric oxidation. All the films were grown on chemically cleaned $\mathrm{Si}(100)$ substrates, which were held at room temperature. A He-Ne laser beam $(633 \mathrm{~nm})$ reflected on the substrate at $(45 \pm 2)^{\circ}$ off the normal was used to record in real time the evolution of the reflectivity of the film as it grew, which allowed monitoring the sample thickness and deposition rate, together with determining an approximate value of the refractive index. The high resolution transmission electron microscopy (HRTEM) analysis reported elsewhere showed that the $\mathrm{Bi}: \mathrm{Ge}$ films are formed by $\mathrm{Bi}$ nanocrystals embedded in amorphous $\mathrm{Ge}(a-\mathrm{Ge})^{14}$ and that the $\mathrm{Al}_{2} \mathrm{O}_{3}$ host is also amorphous. ${ }^{6}$

The ellipsometric parameters, $\tan \Psi$ and $\cos \delta$ were measured by means of a SOPRA spectroscopic rotating polarizer ellipsometer in the 300-800 $\mathrm{nm}$ wavelength range and using steps of $10 \mathrm{~nm}$. The standard deviation of ten measured $\tan \Psi$ and $\cos \delta$ values at the same wavelength for each sample are lower than $5 \times 10^{-4}$ and $10^{-3}$, respectively. The angle of incidence was $(70.00 \pm 0.05)^{\circ}$. The Bruggeman and Maxwell-Garnet effective medium models for threedimensional isotropic systems, considering spherical particles, combined with a standard regression method were used to simulate the measured ellipsometric parameters of the composite films. The optical properties of heterogeneous or composite systems, such as the films analyzed in the present work, have been successfully modeled in the past with the help of effective-medium theories. ${ }^{10}$ In these systems distortions in the microscopic electric field and polarization, due to the mixture of the different materials, give rise to local-field effects and dielectric functions that differ from the simple averages of those of the constituents. Both the Bruggeman and Maxwell-Garnet effective medium models have the same generic expression for an effective medium with two components, $\mathbf{a}$ and $\mathbf{b}$, with dielectric functions $\varepsilon_{a}$ and $\varepsilon_{b}$, and with volume fractions $p_{a}$ and $p_{b}$, respectively: ${ }^{10}$

$$
\frac{\varepsilon_{\mathrm{eff}}-\tilde{\varepsilon}}{\varepsilon_{\mathrm{eff}}+2 \tilde{\varepsilon}}=p_{a} \frac{\varepsilon_{a}-\tilde{\varepsilon}}{\varepsilon_{a}+2 \widetilde{\varepsilon}}+p_{b} \frac{\varepsilon_{b}-\tilde{\varepsilon}}{\varepsilon_{b}+2 \tilde{\varepsilon}},
$$

where $\varepsilon_{\text {eff }}$ is the dielectric functions for the effective medium. The quantity $\tilde{\varepsilon}$ is a dielectric function that is assigned different values according to the model. For MaxwellGarnett model, one of the components is chosen as the host medium, for example, the component $\mathbf{a}$, and then $\tilde{\varepsilon}=\varepsilon_{a}$ and the corresponding term on the right-hand side of Eq. (1) thus vanishes. For the Bruggeman model, the effective medium itself acts as host medium (self-consistent approach) and then $\tilde{\varepsilon}=\varepsilon_{\text {eff }}$, in which case the left-hand side of Eq. (1) vanishes. The resulting calculated spectra for the material systems of our interest show that although both models give similar results for films with a low metal content, the Maxwell-Garnet approximation leads to results which separate from the experimental results for high metal contents. Therefore, results obtained by using the self-consistent Bruggeman approach will only be presented. From Eq. (1) it follows that when the dielectric constants of the two components of the composite are known, and the effective dielectric constant of the medium is measured, then the volume fractions of the components can be determined. Therefore from the SE measurements the Bi content in the films can be obtained. For comparison purposes, the $\mathrm{Bi}$ content in the films was also measured by a standard analytical technique, Rutherford backscattering spectrometry (RBS). The RBS experiments were performed by means of a $2.0 \mathrm{MeV}{ }^{4} \mathrm{He}^{+}$beam and at a scattering angle of $185^{\circ}$ for the $\mathrm{Bi}: \mathrm{Ge}$ films, and by using a $1.0 \mathrm{MeV}{ }^{4} \mathrm{He}^{+}$beam and at a scattering angle of $165^{\circ}$ for the $\mathrm{Bi}: \mathrm{Al}_{2} \mathrm{O}_{3}$ film.

A list of the specimens studied in the present work is shown in Table I, including the most relevant growth parameters and results from the different analytical techniques used. The reported nanocrystals size has been obtained from HRTEM images. ${ }^{14}$ The other results are described and discussed in detail in the following section.

\section{RESULTS AND DISCUSSION}

\section{A. Bi:Ge films}

The Bi:Ge films were grown alternating either 12, 25, 50,100 , or 200 pulses on the Bi target, while keeping a constant number of pulses on the Ge target (100 pulses), and the procedure was repeated five times. The optical constants have been calculated from the ellipsometric parameters assuming an absorbing film on a crystalline silicon substrate and using the thickness determined by RBS for the thinner films (20-40 nm). Figure 1 shows the real $(n)$ and imaginary (k) parts of the refractive index as a function of the wavelength for the films grown with 25 and 200 pulses on $\mathrm{Bi}$ per layer. The $n$ and $k$ values for the films with 12,50 , and 100 pulses of $\mathrm{Bi}$ per layer have not been included in the figure for 
TABLE I. Relevant growth parameters and results from Rutherford backscattering spectrometry (RBS), spectroscopic ellipsometry (SE), and high resolution transmission electron microscopy (HRTEM) measurements, for the pulsed laser deposited films.

\begin{tabular}{|c|c|c|c|c|c|}
\hline \multirow[b]{2}{*}{ Sample } & Growth & \multicolumn{2}{|c|}{$\mathrm{RBS}^{\mathrm{a}, \mathrm{b}}$} & \multirow{2}{*}{$\begin{array}{c}\text { SE } \\
\begin{array}{c}\text { Film thickness } \\
(\mathrm{nm})\end{array}\end{array}$} & \multirow{2}{*}{$\begin{array}{c}\text { HRTEM }^{\mathrm{C}} \\
\text { Bi NCs average } \\
\text { diameter } \\
(\mathrm{nm})\end{array}$} \\
\hline & $\begin{array}{c}\text { per layer on } \mathrm{Bi} \\
\text { target }\end{array}$ & $\begin{array}{c}\mathrm{Bi} \\
\left(\times 10^{15} \mathrm{at} / \mathrm{cm}^{2}\right)\end{array}$ & $\begin{array}{l}\text { Film thickness } \\
(\mathrm{nm})\end{array}$ & & \\
\hline $\mathrm{Bi} 12: \mathrm{Ge}$ & 12 & $6.9 \pm 0.5$ & $20 \pm 2$ & $21 \pm 2$ & $2.3 \pm 0.8$ \\
\hline $\mathrm{Bi} 25$ :Ge & 25 & $12 \pm 1$ & $20 \pm 2$ & $22 \pm 2$ & $2.5 \pm 0.8$ \\
\hline $\mathrm{Bi} 50: \mathrm{Ge}$ & 50 & $24 \pm 2$ & $23 \pm 2$ & $26 \pm 1$ & $7 \pm 2$ \\
\hline $\mathrm{Bi} 100: \mathrm{Ge}$ & 100 & $52 \pm 4$ & $33 \pm 2$ & d & $18 \pm 2$ \\
\hline $\mathrm{Bi} 200: \mathrm{Ge}$ & 200 & $86 \pm 6$ & $44 \pm 3$ & d & $23 \pm 5$ \\
\hline $\mathrm{Al}_{2} \mathrm{O}_{3}$ & 0 & $\ldots$ & $\cdots$ & $236 \pm 2$ & $\ldots$ \\
\hline $\mathrm{Bi}: \mathrm{Al}_{2} \mathrm{O}_{3}$ & 20 & $9.5 \pm 0.7$ & $\cdots$ & $226 \pm 2$ & $<1.5$ \\
\hline
\end{tabular}

${ }^{\mathrm{a}}$ Thicknesses are calculated for the $\mathrm{Bi}: \mathrm{Ge}$ films assuming the $\mathrm{Bi}$ and $\mathrm{Ge}$ bulk densities.

b Thickness for the $\mathrm{Al}_{2} \mathrm{O}_{3}$ and $\mathrm{Bi}: \mathrm{Al}_{2} \mathrm{O}_{3}$ films are not determined, as density of amorphous $\mathrm{Al}_{2} \mathrm{O}_{3}$ could be significantly different than that of the crystalline phases.

${ }^{c}$ Average mean diameter of the Bi nanocrystals from HRTEM images in Ref. 14 for the Bi:Ge films. The quoted errors correspond to the standard deviation of the size distribution. For the $\mathrm{Bi}_{\mathrm{Al}} \mathrm{Al}_{2} \mathrm{O}_{3}$ film, an upper limit for the nanocrystal size is provided according to the resolution limit of the HRTEM for these films (see Ref. 24).

${ }^{\mathrm{d}}$ There is large uncertainty in the thickness determination for these films due to the small penetration length $(<30 \mathrm{~nm})$ of the light in the wavelength measuring range for these samples with high Bi content. Their effective behavior is close to that of a semi-infinite medium, as discussed in Ref. 16.

clarity. For comparison the $n$ and $k$ values for pure PLD $a-\mathrm{Ge}^{15}$ and $\mathrm{Bi}^{16}$ films are included. From the figure it can be observed that while the film grown with 25 pulses on Bi per layer shows $n$ and $k$ values close to those of pure $a$-Ge, the film grown with 200 pulses on Bi per layer has $n$ and $k$ values more similar to those of pure $\mathrm{Bi}$. These results show essentially that the $n$ and $k$ values evolve in a continuous fashion from those of pure $a-\mathrm{Ge}$ to those of pure $\mathrm{Bi}$ as the

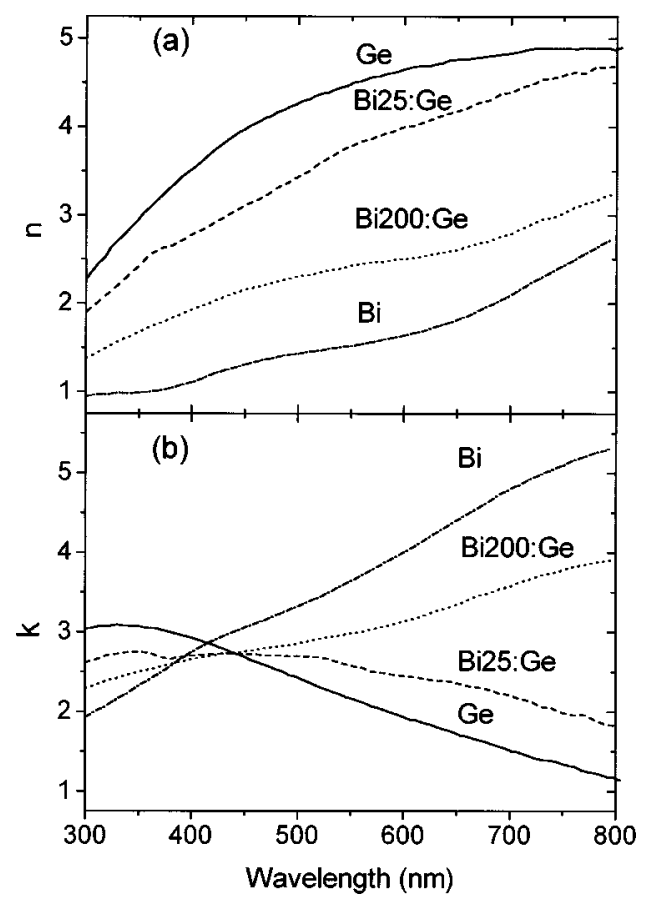

FIG. 1. (a) Real, $n$ and (b) imaginary, $k$, parts of the refractive index as a function of wavelength, for Bi:Ge films grown with 25 and 200 pulses on the Bi target by layer, and for films of the pure elements ( $a-\mathrm{Ge}$ and Bi) (see Refs. 15 and 16) grown by PLD under the same conditions, for reference. number of pulses on Bi per layer increases, and therefore the Bi content in the film or the crystal size ${ }^{14}$ increases.

Figure 2(a) shows the areal density of $\mathrm{Bi}$ atoms as a function of the number of pulses on the Bi target for the Bi:Ge films as determined from the RBS measurements. The

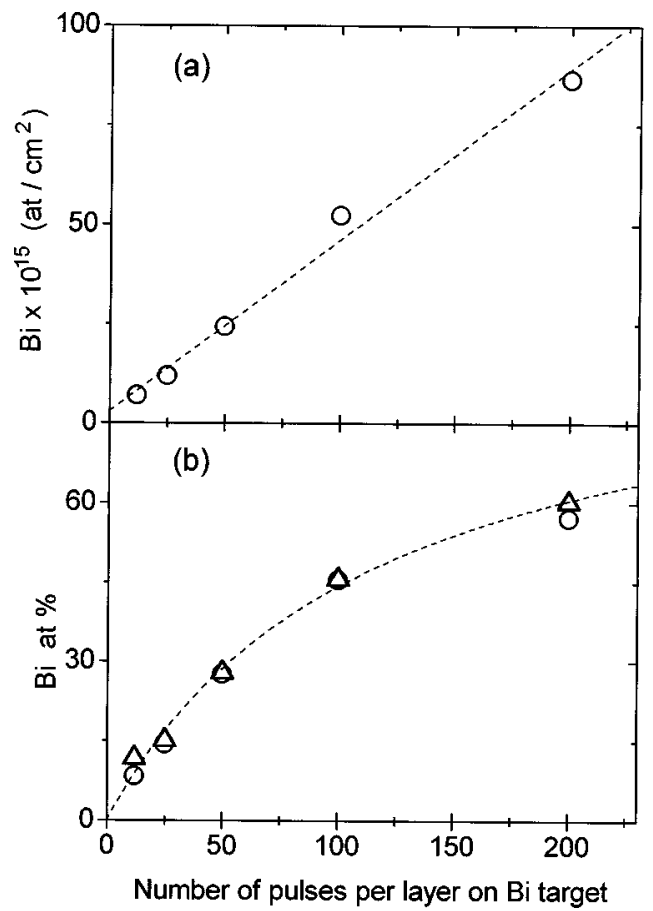

FIG. 2. (a) Areal density of Bi atoms in Bi:Ge films obtained from RBS measurements as a function of the number of pulses used on the Bi target per layer. The dashed line is a linear fit. (b) Bi at. \% of Bi:Ge films as a function of the number of pulses used on the Bi target per layer obtained both from the RBS measurements $(O)$ and from the SE analysis $(\triangle)$. The dashed line is the conversion of the linear fit in (a) to at. \%. The symbol size is larger than the error bar corresponding to the data. 


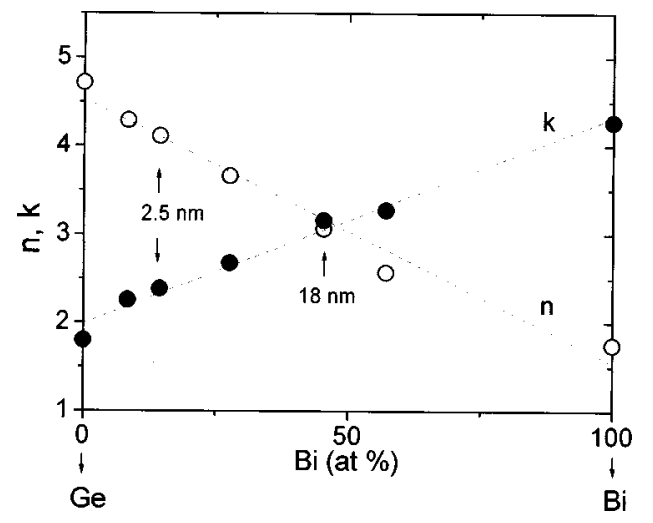

FIG. 3. (○) Real, $n$, and $(\bullet)$ imaginary, $k$, parts of the refractive index of $\mathrm{Bi}$ Ge films at $632.8 \mathrm{~nm}$ as a function of the $\mathrm{Bi}$ at. \%. The values for pure $a$-Ge and Bi films produced by PLD reported elsewhere are included (see Refs. 15 and 16). The dashed lines are linear fits, and the numbers on the plot indicate the average diameter of the Bi nanocrystals for two of the films (see Ref. 14). The symbol size is larger than the error bar corresponding to the data.

areal density of $\mathrm{Bi}$ follows a linear dependence with the number of pulses, thus indicating a good control of the experimental conditions, which allows to vary very accurately the amount of metal atoms. From these data it can be calculated that around $9 \times 10^{13} \mathrm{Bi}$ at $/ \mathrm{cm}^{2}$ are only deposited on the substrate with a single pulse. The areal density of the deposited Ge is $6.7 \pm 0.5 \times 10^{16} \mathrm{at} / \mathrm{cm}^{2}$ which is the same for all the films within the experimental error. Taking into account both $\mathrm{Bi}$ and $\mathrm{Ge}$ areal densities, the $\mathrm{Bi}$ at. \% of the films has been calculated, and it is shown in Fig. 2(b). From the areal density data obtained from the RBS measurements, the total thickness of the film was estimated by assuming that the density of the deposited materials are those of bulk Bi (2.8 $\left.\times 10^{22} \mathrm{at} / \mathrm{cm}^{3}\right)$ and $\mathrm{Ge}\left(4.4 \times 10^{22} \mathrm{at} / \mathrm{cm}^{3}\right)$. The film thickness increases with the amount of $\mathrm{Bi}$, from $20 \mathrm{~nm}$ for the thinnest film (12 pulses on $\mathrm{Bi}$ ) to $44 \mathrm{~nm}$ for the thickest film (200 pulses on $\mathrm{Bi}$ ).

The Bruggeman effective medium model has been used to simulate the measured ellipsometric parameters of the films, by assuming a three-dimensional isotropic system formed by $a-\mathrm{Ge}$ and Bi with the optical parameters ( $n$ and $k$ ) reported earlier for PLD films of the pure elements. ${ }^{15,16}$ The effective medium model through the use of a standard regression method gives the volume fraction of $\mathrm{Bi}$ in $a-\mathrm{Ge}$, and the film thickness which leads to the best fit. Good fits to the experimental data were obtained following this procedure, with an unbiased estimator always lower than 0.02. ${ }^{15}$ The composition of the films in at. \% has been calculated from the Bi volume fraction assuming again the density of the bulk materials given above, and the results are also plotted in Fig. 2(b). It is clearly seen that the compositions determined by SE and RBS measurements are in excellent agreement.

To illustrate further the continuous evolution of the optical properties of the composite from those of the matrix to those of the metal, the real $(n)$ and imaginary $(k)$ parts of the refractive index $\mathbf{n}$ for the Bi:Ge films are plotted in Fig. 3 as a function of the $\mathrm{Bi}$ at. \% for one of the most widely used laser wavelength $(632.8 \mathrm{~nm})$. The data have been inter- polated between the data acquired at 630 and $640 \mathrm{~nm}$. The results show that both components of the refractive index follow a linear dependence with the metal content in a first approximation. The HRTEM images reported elsewhere ${ }^{14}$ show that the films are formed by well separated Bi nanocrystals embedded in the $a$-Ge matrix. The average diameter of the nanocrystals for the different samples is summarized in Table I, and it is also included for two representative films in the figure. It is clearly seen that the linear dependence of the optical constants of the composite film on the metal content even applies for crystal sizes above $18 \mathrm{~nm}$. Furthermore, for the films with the higher metal content in which the metal layer is quite close to a continuous layer, the results plotted both in Figs. 2 and 3 show that the Bruggeman model still applies and provides a consistent simulation of the SE parameters.

\section{B. $\mathrm{Bi}: \mathrm{Al}_{2} \mathrm{O}_{3}$ film}

The $\mathrm{Bi}: \mathrm{Al}_{2} \mathrm{O}_{3}$ film was grown alternating 20 pulses on the $\mathrm{Bi}$ target and 1900 pulses on the $\mathrm{Al}_{2} \mathrm{O}_{3}$ target, and repeating the procedure ten times. As a reference an $\mathrm{Al}_{2} \mathrm{O}_{3}$ film was produced in the same conditions but with no $\mathrm{Bi}$ nanocrystals. Therefore the $\mathrm{Al}_{2} \mathrm{O}_{3}$ film was grown with a total of 19000 pulses on the $\mathrm{Al}_{2} \mathrm{O}_{3}$ target.

In order to analyze the optical constants of the nanocomposite $\mathrm{Bi}: \mathrm{Al}_{2} \mathrm{O}_{3}$ following the same scheme as that used for the $\mathrm{Bi}: \mathrm{Ge}$ films it is necessary as a first step to determine the optical constants of the $\mathrm{Al}_{2} \mathrm{O}_{3}$ film grown by PLD. To our knowledge, there are no earlier reports of the optical constants of amorphous $\mathrm{Al}_{2} \mathrm{O}_{3}$ films grown by PLD over the wavelength range of our interest. Therefore the SE parameters for the $\mathrm{Al}_{2} \mathrm{O}_{3}$ with no $\mathrm{Bi}$ nanocrystals were measured and are shown in Figs. 4(a) and 4(b). Since thickness and refractive index are cross-related values in ellipsometry, a Cauchy function was first assumed for the real part of the refractive index of the film in order to obtain a consistent approach for the film thickness. In this way it is assured that $n$ is well behaved (i.e., it is smooth and follows a negative dispersion law). Thus the expression

$$
n_{c}(\lambda)=A+\frac{B}{\lambda^{2}}+\frac{C}{\lambda^{4}}
$$

for the real part of the refractive index was used to fit the SE data, assuming a nonabsorbing film $(k=0)$ and with $\lambda$ being the wavelength. Figures 4(a) and 4(b) also include the best fit of the experimental data which was achieved with a thickness of $236 \pm 2 \mathrm{~nm}$ and parameters $A=1.638, \quad B$ $=0.0058 \mu \mathrm{m}^{-2}$, and $C=0.0002 \mu \mathrm{m}^{-4}$. The corresponding results for the $n_{\mathrm{c}}$ values are shown in Fig. 4(c). Correlation coefficients among the fitting parameters are quite low, except for the case of the correlation coefficient for thickness and parameter A which is 0.88 . Statistical errors for $n_{c}$ are lower than 0.01 . Using the thickness obtained from this fitting, the $n$ and $k$ values were calculated from direct numerical inversion, and the results are also shown in Fig. 4(c). The obtained $n$ values show a fairly smooth dependence as a function of $\lambda$, which follows a negative dispersion law as it does for sapphire (bulk crystalline $\left.\mathrm{Al}_{2} \mathrm{O}_{3}\right) \cdot{ }^{17}$ Nevertheless, 


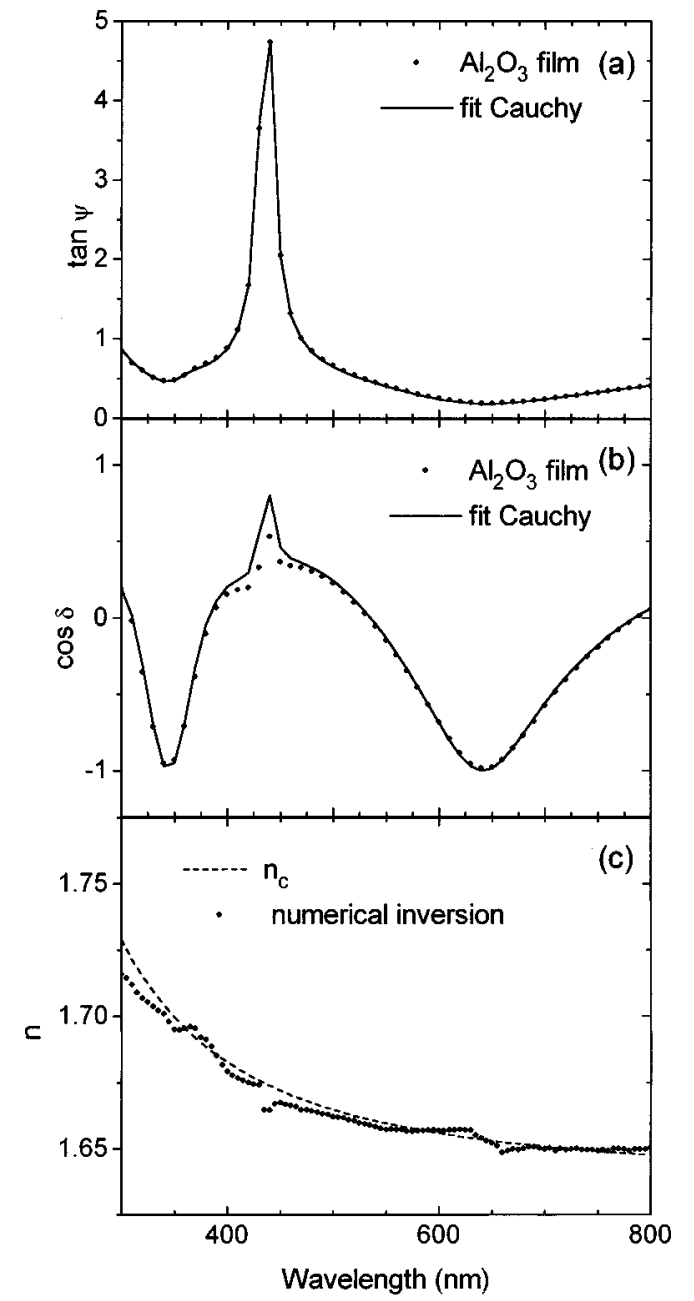

FIG. 4. (a) Tan $\Psi$ and (b) $\cos \delta$ measured values for the $\mathrm{Al}_{2} \mathrm{O}_{3}$ film with no Bi nanocrystals (dotted line) and their best fit assuming a nonabsorbing film with the real part of the refractive index represented by a Cauchy function (solid line). (c) Real part of the refractive index for the $\mathrm{Al}_{2} \mathrm{O}_{3}$ film with no $\mathrm{Bi}$ nanocrystals from the fits in (a) and (b) (dashed line), and from direct inversion of the experimental SE data (dotted line).

three small discontinuities in the $n$ spectrum can be observed at the wavelengths at which $\delta$ approaches $0^{\circ}$ or $180^{\circ}$ $(\cos \delta= \pm 1)$. They are artificial structures originated due to interference effects in thin films which have no physical meaning and thus are artifacts. The obtained $\mathrm{k}$ values from direct inversion are negligible in the whole studied wavelength range showing consistency with the assumption used.

Figure 5(a) shows the in situ measured evolution of the reflectivity at $632.8 \mathrm{~nm}$ of the $\mathrm{Bi}: \mathrm{Al}_{2} \mathrm{O}_{3}$ film during growth and as a function of time. The initial reflectivity corresponds to the $\mathrm{Si}$ substrate reflectivity measured at $(45 \pm 2)^{\circ}$. This value has been used to normalize the experimental values and to refer the data to an absolute reflectivity scale. The reflectivity shows a maximum value at the beginning $(\mathrm{Si}$ substrate reflectivity), goes through a minimum at about $2000 \mathrm{~s}$ deposition time and finally reaches a maximum at about $4000 \mathrm{~s}$ deposition time. The reflectivity of a homogeneous dielectric film on $\mathrm{Si}$ as a function of the thickness can be easily calculated, ${ }^{18}$ using the values reported elsewhere for the crystalline silicon substrate. ${ }^{19}$ The $n$ and $k$ values for

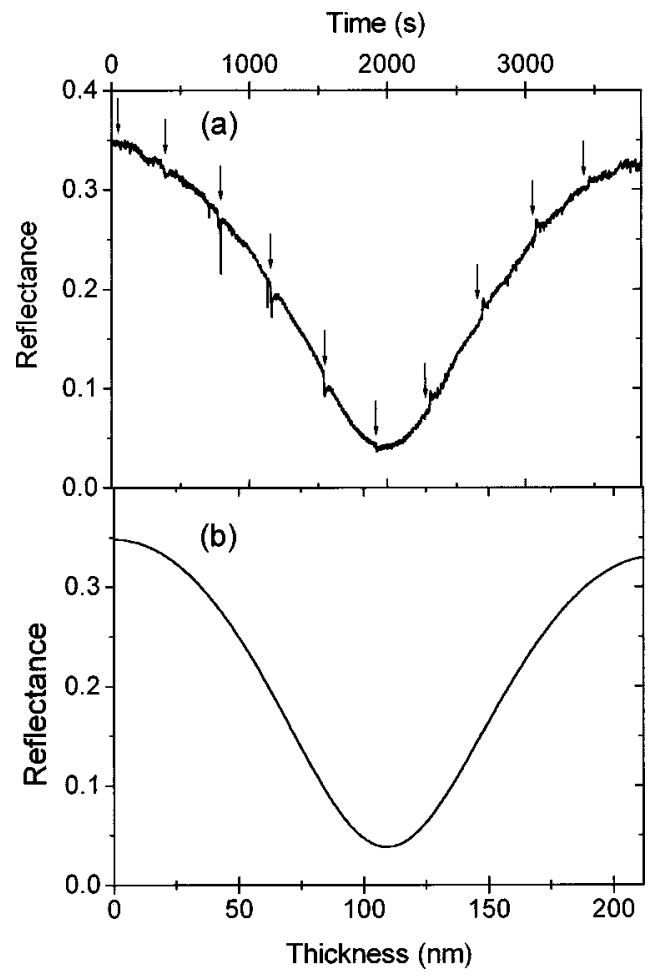

FIG. 5. (a) Evolution of the reflectivity at $632.8 \mathrm{~nm}$ of the $\mathrm{Bi}^{\mathrm{A}} \mathrm{Al}_{2} \mathrm{O}_{3}$ film during deposition as a function of time. The arrows in the plot $(\downarrow)$ mark the point at which Bi deposition starts. (b) Simulation of the reflectance at $45^{\circ}$ for an $\mathrm{Al}_{2} \mathrm{O}_{3}$ film growing on a $\mathrm{Si}$ substrate as a function of thickness.

sapphire (crystalline $\left.\mathrm{Al}_{2} \mathrm{O}_{3}\right)^{17}$ were initially used for the calculation, and then modified until the best fit for the experimental results in Fig. 5(a) was obtained. The result of this calculation is shown in Fig. 5(b), which was obtained with $n=1.620 \pm 0.005, k=0.010 \pm 0.005$, and a thickness of 210 $\pm 10 \mathrm{~nm}$.

The essential feature which differences the experimental [Fig. 5(a)] and simulated [Fig. 5(b)] curves are the small transient fluctuations (negative peaks for time $<2000 \mathrm{~s}$, or positive peaks for time $>2000 \mathrm{~s}$ ) which appear over the smooth U-shaped curve always a few seconds after the Bi deposition has been initiated (arrows in the figure), and disappear as soon as enough $\mathrm{Al}_{2} \mathrm{O}_{3}$ is subsequently deposited. We have simulated the reflectivity of a $\mathrm{Bi}$ layer (high reflectivity) growing on the low-absorbing $\mathrm{Al}_{2} \mathrm{O}_{3}$ layer and the result shows a transient enhancement of the reflectivity for time $<2000 \mathrm{~s}$ and a transient decrease of the reflectivity for time $>2000 \mathrm{~s}$ as opposed to what is observed experimentally in Fig. 5(a). Therefore, the experimentally observed peaks are most likely related to the appearance of surface roughness as the $\mathrm{Bi}$ nanocrystals develop at the surface of the $\mathrm{Al}_{2} \mathrm{O}_{3}$ layer and are subsequently buried. ${ }^{20}$ This is indeed an in situ indication that metallic $\mathrm{Bi}$ nanocrystals are formed on the $\mathrm{Al}_{2} \mathrm{O}_{3}$.

Figures 6(a) and 6(b) shows the $\tan \Psi$ and $\cos \delta$ values measured for the $\mathrm{Bi}: \mathrm{Al}_{2} \mathrm{O}_{3}$ film. Since the optical parameters of the two components of the film, $\mathrm{Bi}^{16}$ and $\mathrm{Al}_{2} \mathrm{O}_{3}$ [from Fig. 4(c)] are known, the effective medium model is applied to determine the thickness of the composite film, the best fit being obtained for $226 \pm 2 \mathrm{~nm}$ which is in good agreement 


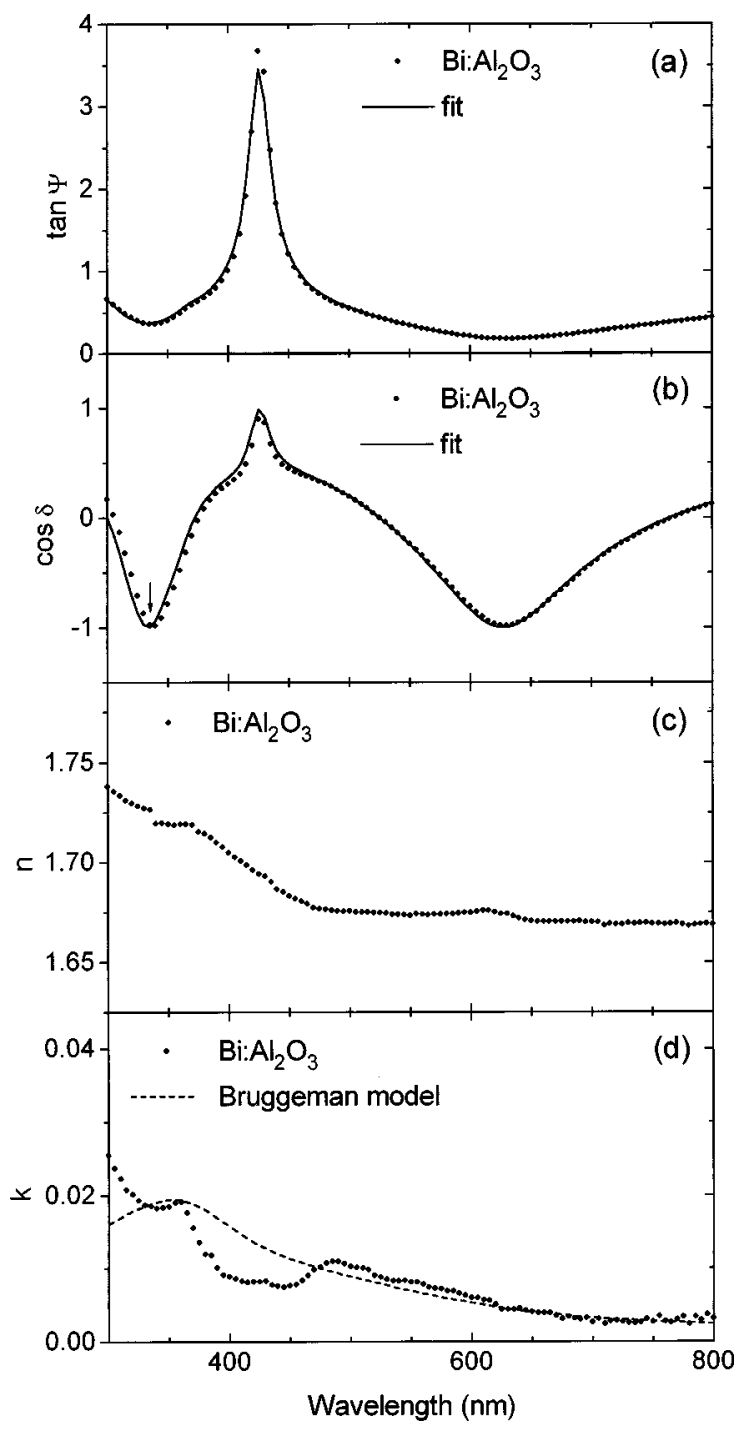

FIG. 6. (a) Tan $\Psi$ and (b) $\cos \delta$ measured values for the $\mathrm{Bi} \mathrm{Al}_{2} \mathrm{O}_{3}$ film (dotted line) and their best fit. (c) Real, $n$, and (d) imaginary, $k$, parts of the refractive index of the $\mathrm{Bi}: \mathrm{Al}_{2} \mathrm{O}_{3}$ film as a function of wavelength. The $k$ evolution for a $\mathrm{Bi}: \mathrm{Al}_{2} \mathrm{O}_{3}$ film with a 0.14 at. $\%$ of $\mathrm{Bi}$ calculated using the Bruggeman effective medium model is also included in (d) (dashed line).

with the value obtained independently from the in situ reflectivity measurements (Fig. 5), and compares very well with the value determined for the $\mathrm{Al}_{2} \mathrm{O}_{3}$ with no $\mathrm{Bi}$ nanocrystals grown in the same conditions. This thickness value is finally used to determine the refractive index $(\mathbf{n}=n+i k)$ of the $\mathrm{Bi}: \mathrm{Al}_{2} \mathrm{O}_{3}$ film by numerical inversion, and its components are shown in Figs. 6(c) and 6(d). The comparison of Fig. 4(c) and Fig. 6(c) evidences that the real part of the refractive index shows the same general trend for the $\mathrm{Al}_{2} \mathrm{O}_{3}$ and $\mathrm{Bi}: \mathrm{Al}_{2} \mathrm{O}_{3}$ films, and it is slightly higher for the latter film. Besides, the comparison of the components of the refractive index in Fig. 6 in the wavelength range from $630-640 \mathrm{~nm}$ to the values obtained from the simulation of the in situ reflectivity measurements at $632.8 \mathrm{~nm}$ (Fig. 5) evidences that the latter method gives an independent and good approximation to the optical constants of the film. Although the real part of the refractive index values for the $\mathrm{Al}_{2} \mathrm{O}_{3}$ film with and without $\mathrm{Bi}$ nanocrystals are very similar, the imaginary part of the refractive index is, in contrast, quite different for the two films. For the $\mathrm{Al}_{2} \mathrm{O}_{3}$ film with no $\mathrm{Bi}$ nanocrystals, the linear absorption is negligible $(k<0.001)$ in the whole studied wavelength range. However, for the film containing $\mathrm{Bi}$ nanocrystals the $k$ value increases from 0.003 at $800 \mathrm{~nm}$ to 0.025 at $300 \mathrm{~nm}$ [Fig. 6(d)]. Furthermore, some structure is observed in the $\mathrm{k}$ spectrum including two broad peaks, one at around $360 \mathrm{~nm}$ and the other at $500 \mathrm{~nm}$. The increase of the absorption has to be related to the inclusion of the $\mathrm{Bi}$ in the film. Regarding the structure of the $k$ and $n$ spectra it must be noted that some of its features can be originated by the limitations of the measurement system when $\delta$ approaches $0^{\circ}$ or $180^{\circ}(\cos \delta= \pm 1)$, similarly to what has been discussed for the $\mathrm{Al}_{2} \mathrm{O}_{3}$ film. This is the case of the discontinuities in both the $n$ and $k$ spectra at 325, 425, and $625 \mathrm{~nm}$. Nevertheless, the two broad peaks observed in the $k$ spectrum are far enough from these wavelengths and cannot be explained as a consequence of wrong ellipsometric analysis. It should be noted that there has to be a physical effect giving rise to the appearance of these broad features as a consequence of the inclusion of metal nanosized particles in the dielectric film.

Although effective medium theories such as Bruggeman or Maxwell-Garnett models provide the best approach to analyze the optical properties of metal-dielectric composites, ${ }^{7,8}$ a qualitative understanding can be obtained as a first step within the frame of the more simple Mie theory for the diffraction of a conducting sphere in the electric dipole approximation. ${ }^{21,22}$ In this approach it is imposed the vanishing of the forward scattering amplitude and all the terms of higher order than the dipolar one are neglected. ${ }^{1}$ For the case of a dielectric containing metal nanoparticles with characteristic sizes less than the wavelength, the extinction coefficient $k$ can be described as

$$
k=\frac{9 p n_{d}^{3}}{2} \frac{\varepsilon_{2}}{\left(\varepsilon_{1}+2 n_{d}^{2}\right)^{2}+\varepsilon_{2}^{2}},
$$

where $p$ is the metal volume fraction, $\varepsilon=\varepsilon_{1}+i \varepsilon_{2}$ is the dielectric constant of the metal, and $n_{d}$ is the index of refraction of the dielectric host media. According to this expression the absorption is expected to exhibit an enhancement where the condition $\varepsilon_{1}+2 n_{d}^{2}=0$ is met, the surface plasmon resonance frequency (SPR). Using the data in Fig. 1 for the metal and in Fig. 4(c) for the host, it can be seen that this condition is satisfied for a wavelength between 370 and 380 $\mathrm{nm}$, quite close to the wavelength at which $k$ determined from the SE measurements [Fig. 6(d)] reaches a maximum. It should be noted that the SPR is a characteristic for metal nanocrystals embedded in a dielectric (nonabsorbing) host, and it is not found for the case of a semiconducting (absorbing) host as $a-\mathrm{Ge}$, since the absorbing nature of the host prevents the occurrence of confinement effects. In the case of the dielectric host, the confinement effects become apparent due to the fact that the size of the nanocrystals is much smaller than the measuring wavelength and their dielectric constant is very different than that of the surrounding non absorbing dielectric. Thus, the electric field that acts on and polarizes the charges of these nanocrystals can be vastly different from the macroscopic Maxwell field. ${ }^{1}$ The slight dif- 
ferences in the dielectric constants of the nanocrystals and the host in the Bi:Ge system within the studied wavelength range attenuate the existence of possible confinement effects.

A deeper approach to the problem can be performed through the simulation of the SE measured parameters using the Bruggemans effective medium model. The best fit is obtained assuming a mixture of $\mathrm{Al}_{2} \mathrm{O}_{3}$ with $0.45 \% \mathrm{Bi}$ in volume. Figure 6(d) includes the calculated $\mathrm{k}$ value for such a mixture which also shows a maximum at around $360 \mathrm{~nm}$, consistent with both the calculated $\mathrm{SPR}$ of $\mathrm{Bi}$ in $\mathrm{Al}_{2} \mathrm{O}_{3}$ from Eq. (3) and the experimental data, and supports further that this peak is related to the presence of Bi nanocrystals in the film. The experimental results in Fig. 6(d) also show a second absorption peak around $500 \mathrm{~nm}$ which is not reproduced by the simulation. Although its origin is still unclear, it might be related to interference, roughness or inhomogeneities at the film-silicon substrate interface or at the film surface.

Regarding the determination of the $\mathrm{Bi}$ concentration in the $\mathrm{Bi}: \mathrm{Al}_{2} \mathrm{O}_{3}$ film, the simulation of the SE parameters measured for the $\mathrm{Bi}: \mathrm{Al}_{2} \mathrm{O}_{3}$ film with the Bruggeman effective medium model leads to the best fit for a $0.45 \%$ of $\mathrm{Bi}$ in volume. On the other hand, the RBS measurements performed in the $\mathrm{Bi}: \mathrm{Al}_{2} \mathrm{O}_{3}$ film give an areal density of (1.9 $\pm 0.2) \times 10^{18} \mathrm{at} / \mathrm{cm}^{2}$ for the $\mathrm{Al}_{2} \mathrm{O}_{3}$ and $(9.5 \pm 0.5)$ $\times 10^{15} \mathrm{at} / \mathrm{cm}^{2}$ for the $\mathrm{Bi}$, which leads to a concentration of 0.5 at. \% $\mathrm{Bi}$. Both results on the $\mathrm{Bi}$ concentration can only be compared if the density of the amorphous $\mathrm{Al}_{2} \mathrm{O}_{3}$ is first determined. This can be easily done taking into account the film thickness of the $\mathrm{Al}_{2} \mathrm{O}_{3}$ film with no $\mathrm{Bi}$ nanocrystals obtained by SE $(236 \pm 2 \mathrm{~nm})$ and the corresponding areal density measured by RBS for this film (2.0 \pm 0.2$)$ $\times 10^{18} \mathrm{at} / \mathrm{cm}^{2}$. The results leads to a density of $(8.7 \pm 0.8)$ $\times 10^{22} \mathrm{at} / \mathrm{cm}^{3}$ which is lower than that of sapphire (crystalline $\left.\mathrm{Al}_{2} \mathrm{O}_{3}\right) .{ }^{17}$ The lower density is consistent with the result that the measured real part of the refractive index $[n$ value in Fig. 4(c)] is also lower than that of sapphire. The LorentzLorenz equation links the refractive index of the dielectric $n_{d}$ directly to the density $\rho:^{23}$

$$
\frac{\left(n_{d}^{2}-1\right)}{\left(n_{d}^{2}+2\right)}=\frac{4 \pi R \rho}{3 M},
$$

where $R$ is the molar polarizability and $M$ the molar weight. According to this formulation, a decrease in the density of the media leads to a decrease in the refractive index. Since in our case the lower refractive index cannot be fully explained by the lower density of the films, changes in the molar polarizability of the amorphous films in respect to sapphire have also to be taken into account.

Finally, assuming the calculated density for the amorphous $\mathrm{Al}_{2} \mathrm{O}_{3}$ film and that of bulk $\mathrm{Bi}$, the volume fraction of Bi calculated from the SE measurements, $0.45 \%$, becomes a $\mathrm{Bi}$ atomic fraction of 0.14 at. \%. Even though the absolute difference between the $\mathrm{Bi}$ at. \% obtained from SE and RBS measurements may seem small, 0.14 and 0.5 at. \%, respectively, unacceptable large $k$ values are obtained for a mixture with 0.5 at. \% Bi. It is then clear that SE underestimates the $\mathrm{Bi}$ content in the $\mathrm{Bi}: \mathrm{Al}_{2} \mathrm{O}_{3}$ films as opposed to the excellent agreement obtained in the case of $\mathrm{Bi}: G e$ films. One possible explanation is that in the $\mathrm{Bi}: \mathrm{Al}_{2} \mathrm{O}_{3}$ case, the deposited $\mathrm{Bi}$ is surrounded by a shell of oxygen atoms coming from the dielectric matrix that may alter the homogeneous optical properties assumed for $\mathrm{Bi}$ and $\mathrm{Al}_{2} \mathrm{O}_{3}$ and this effect should be larger the smaller the Bi crystals. Further indication that this mechanism might be very important for our films relies in the fact that the nanocrystals could not be observed for this film by HRTEM, which is consistent with nanocrystals with size smaller than $1.5 \mathrm{~nm} .{ }^{24}$ Interface processes resulting from the interaction of the nanoparticles with the surrounding media, such as that mentioned above, have been reported to influence drastically the optical spectra, ${ }^{25,26}$ and would be most important for these small crystals in which the surface to volume ratio is large.

\section{CONCLUSIONS}

The results presented here evidence that SE together with the effective medium model is a very suitable means to determine simultaneously the effective refractive index, thickness, and metal content of thin nanocomposite films. The method works very well in the case of non-miscible systems (Bi:Ge films) and it leads to an underestimation of the metal content in the case of the oxide host $\left(\mathrm{Bi}: \mathrm{Al}_{2} \mathrm{O}_{3}\right.$ films) with very small nanocrystals $(<1.5 \mathrm{~nm})$ probably due to some interaction between the metal and the host. In the case of an absorbing host (Bi:Ge films), both the real and imaginary parts of the refractive index follow a linear behavior from that of the host to that of the metal as the amount of metal increases. In the case of the nonabsorbing host, $\mathrm{Al}_{2} \mathrm{O}_{3}$ films, the SE allows to determine the film thickness, and taking into account the RBS data for the number of atoms per area unit, the density of the PLD amorphous $\mathrm{Al}_{2} \mathrm{O}_{3}$ film has been determined, resulting lower than that of sapphire. The real part of the refractive index of the $\mathrm{Bi}_{\mathrm{Al}} \mathrm{Al}_{2} \mathrm{O}_{3}$ films is slightly higher than that of the host and the imaginary part shows a characteristic absorption peak at a wavelength (360 $\mathrm{nm}$ ) very close to that predicted for the surface plasmon resonance.

\section{ACKNOWLEDGMENTS}

This work has been partially supported by CICYT (Spain) under TIC96-0467 project. The authors are grateful to the GPS (Université de Paris VI et VII, France) for provision and assistance of Rutherford backscattering facilities. One of the authors (J.M.B.) greatly acknowledges a FPI grant from the Spanish Ministry of Education and Culture. Dr. E. Bernabeu (UCM, Spain) is thanked for provision of spectroscopic ellipsometry facilities.

${ }^{1}$ C. Flytzanis, F. Hache, M. C. Kelin, D. Ricard, and Ph. Roussignol, in Progress in Optics, edited by E. Wolf (North-Holland, Amsterdam, 1991), Vol. XXIX, pp. 323-411.

${ }^{2}$ E. M. Vogel, M. J. Weber, and D. M. Krol, Phys. Chem. Glasses 32, 231 (1991).

${ }^{3}$ Z. Pan, S. H. Morgan, D. O. Henderson, S. Y. Park, R. A. Weeks, R. H. Magruder III, and R. A. Zuhr, Opt. Mater. 4, 675 (1995).

${ }^{4}$ S. Ohtsuka, K. Tsunetomo, T. Koyama, and S. Tanaka, Opt. Mater. 2, 209 (1993).

${ }^{5}$ T. Yoshida, S. Takeyama, Y. Yamada, and K. Mutoh, Appl. Phys. Lett. 68, 1772 (1996).

${ }^{6}$ J. M. Ballesteros, R. Serna, J. Solis, C. N. Afonso, A. K. Petford-Long, D. H. Osborne, and R. F. Haglund, Jr., Appl. Phys. Lett. 71, 2445 (1997). 
${ }^{7}$ R. W. Cohen, G. D. Cody, M. D. Coutts, and B. Abeles, Phys. Rev. B 8, 3689 (1973).

${ }^{8}$ T. Yamaguchi, M. Sakai, and N. Saito, Phys. Rev. B 32, 2126 (1985).

${ }^{9}$ P. Tognini, M. Gedo, A. Stella, P. Cheysac, and R. Kofman, J. Appl. Phys. 79, 1032 (1996).

${ }^{10}$ D. E. Aspnes, in Handbook of Optical Constants of Solids, edited by E. D. Palik (Academic, Orlando, FL, 1985), Chap. 5, pp. 89-112.

${ }^{11}$ F. Vega, J. C. G. de Sande, C. N. Afonso, C. Ortega, and J. Siejka, Appl. Opt. 33, 1203 (1994).

${ }^{12}$ J. C. de Sande, F. Vega, C. N. Afonso, C. Ortega, and J. Siejka, Thin Solid Films 249, 195 (1994).

${ }^{13}$ T. Missana, C. N. Afonso, and M. F. da Silva, Appl. Phys. A 59, 653 (1994).

${ }^{14}$ R. Serna, T. Missana, C. N. Afonso, J. M. Ballesteros, A. K. PetfordLong, and R. C. Doole, Appl. Phys. A 66, 43 (1998).

${ }^{15}$ J. C. G. de Sande, C. N. Afonso, J. L. Escudero, R. Serna, F. Catalina, and E. Bernabeu, Appl. Opt. 31, 6133 (1992).

${ }^{16}$ J. C. G. de Sande, T. Missana, and C. N. Afonso, J. Appl. Phys. 80, 7023 (1996).
${ }^{17}$ Handbook of Infrared Optical Materials, edited by P. Klocek (Marcel Dekker, Inc., New York, 1991) p. 196.

${ }^{18}$ M. Born and E. Wolf, Principles of Optics (Pergamon, Oxford, 1983), Chap. 1, p. 61.

${ }^{19}$ D. F. Edwards, in Handbook of Optical Constants of Solids, edited by E. D. Palik (Academic, Orlando, FL, 1985), Subpart 2, pp. 552-570.

${ }^{20}$ C. D. Zuiker, D. M. Gruen, and A. R. Krauss, MRS Bull. 20, 29 (1995).

${ }^{21}$ G. Mie, Ann. Phys. (Leipzig) 25, 377 (1908).

${ }^{22}$ M. Born and E. Wolf, Principles of Optics (Pergamon, Oxford, 1983), Chap. 13, pp. 633-667.

${ }^{23}$ M. Born and E. Wolf, Principles of Optics (Pergamon, Oxford, 1983), Chap. 2, p. 87.

${ }^{24}$ C. N. Afonso, R. Serna, J. M. Ballesteros, A. K. Petford-Long, and R. C. Doole, Appl. Surf. Sci. 127-129, 339 (1998).

${ }^{25} \mathrm{U}$. Kreibig and M. Vollmer, Optical Properties of Metal Clusters (Springer, Berlin, 1995), Chap. 4, pp. 380-387.

${ }^{26}$ T. Brandt, W. Hoheisel, A. Iline, F. Stietz, and F. Träger, Appl. Phys. B 65, 793 (1997). 